Short-Term Benefits of an Unrestricted-Calorie Traditional Mediterranean Diet, Modified With a Reduced Consumption of Carbohydrates at Evening, in Overweight-Obese Patients.

Roberto Salvia ${ }^{1,2}$, Simona D’Amore ${ }^{3}$, Giusi Graziano ${ }^{3}$, Caterina Capobianco ${ }^{1}$, Moris Sangineto ${ }^{2}$, Domenico Paparella ${ }^{1}$, Paola De Bonfils ${ }^{1}$, Giuseppe Palasciano ${ }^{1}$, Michele Vacca ${ }^{1,2}$.

1 Clinica Medica “Augusto Murri”, University of Bari “Aldo Moro”, 70124 Bari, Italy; ${ }^{2}$ Dipartimento Interdisciplinare di Medicina, University of Bari “Aldo Moro”, 70124 Bari, Italy; ${ }^{3}$ National Cancer Research Centre, IRCCS Istituto Oncologico Giovanni Paolo II, 70124 Bari, Italy. 
Salvia et al., Online Supporting Materials

Supplemental Table 1. Adherence to Mediterranean Diet in the study population before

\section{nutritional intervention.}

\begin{tabular}{|l|c|c|c|c|c|c|c|}
\hline & \multicolumn{7}{|c|}{ Legend } \\
\hline ITEMS & MEAN \pm SEM & Never & $1-4$ & $5-8$ & $9-12$ & $13-18$ & $>18$ \\
\hline Full fat dairy products & $1.3 \pm 0.3$ & 5 & 4 & 3 & 2 & 1 & 0 \\
\hline Red meat and meat products & $1.4 \pm 0.3$ & 5 & 4 & 3 & 2 & 1 & 0 \\
\hline Poultry & $2.5 \pm 0.3$ & 5 & 4 & 3 & 2 & 1 & 0 \\
\hline Potatoes & $1.2 \pm 0.2$ & 0 & 1 & 2 & 3 & 4 & 5 \\
\hline Non-refined cereals & $1.6 \pm 0.4$ & 0 & 1 & 2 & 3 & 4 & 5 \\
\hline Vegetables & $4.6 \pm 0.2$ & 0 & 1 & 2 & 3 & 4 & 5 \\
\hline Fruits & $4.8 \pm 0.2$ & 0 & 1 & 2 & 3 & 4 & 5 \\
\hline Legumes & $2.4 \pm 0.3$ & 0 & 1 & 2 & 3 & 4 & 5 \\
\hline Fish & $2.2 \pm 0.3$ & 0 & 1 & 2 & 3 & 4 & 5 \\
\hline Olive oil (times/week) & MEAN \pm SEM & Never & Rare & $<1$ & $1-3$ & $3-5$ & Daily \\
\hline & $5.0 \pm 0.0$ & 0 & 1 & 2 & 3 & 4 & 5 \\
\hline Alcoholic beverages (ml/day) & MEAN \pm SEM & $<300$ & 300 & 400 & 500 & 600 & $>700$ or 0 \\
\hline \multicolumn{2}{|c|}{$4.5 \pm 0.2$} & 5 & 4 & 3 & 2 & 1 & 0 \\
\hline Total & $\mathbf{3 3 . 9 \pm 0 . 3}$ & & & & & \\
\hline
\end{tabular}

Data are reported as a means \pm SEM. Eleven main components of the Mediterranean diet (nonrefined cereals, fruits, vegetables, potatoes, legumes, olive oil, fish, red meat, poultry, full fat dairy products and alcohol) were considered. In detail, we used monotonic functions (with the exception of alcohol intake) in order to score the frequency consumption of these foods. In particular, we assigned individual ratings (from 0 to 5 or the reverse) in each of the 11 food groups according to their position in the Mediterranean diet pyramid. For the consumption of items presumed to be close to this pattern (non-refined cereals, fruits, vegetables, legumes, olive oil, fish, and potatoes) we assigned score 0 when someone reported no consumption, score 1 when they reported consumption of 1-4 servings/month, score 2 for 5-8 servings/month, score 3 for 9-12 servings/month, score 4 for 13-18 servings/month and score 5 for more than 18 servings/month. On the other hand, for the consumption of foods presumed to be outside of this diet (meat and meat products, poultry and full fat dairy products) we assigned the scores on a reverse scale (i.e. 5 when someone reported no consumption to 0 when they reported almost daily consumption). For olive oil we evaluated its weekly use for cooking and we assigned 0 when no consumption was reported, 1 for rare consumption, 2 for less than 1 times a week, 3 for 1-3 times a week, 4 for 3-5 times a week and 5 for daily use. For alcohol we did not use a monotonic function, but we assigned score 5 for consumption of less than $300 \mathrm{ml}$ of alcohol/day, score 0 for consumption of more than 700 (or 0 ) $\mathrm{ml} /$ day and scores from 4 to 1 for daily consumption of 300, 400, 500 and $600 \mathrm{ml}(100 \mathrm{ml}$ has $12 \mathrm{~g}$ ethanol concentration), respectively (Panagiotakos et al., 2006). 
Supplemental Table 2: List of food to minimize or avoid in the evening.

\begin{tabular}{|l|l|}
\hline Cereals grains & $\begin{array}{l}\text { Corn, Cornmeal, Couscous, Whole wheat, White rice, } \\
\text { Semolina. }\end{array}$ \\
\hline Breads & $\begin{array}{l}\text { Wheat Breads, White wheat flour bread, Fiber-enriched, } \\
\text { White bread, Specialty wheat breads (pita bread), Rice bread, } \\
\text { Grissini, Taralli, Crackers. }\end{array}$ \\
\hline Pasta/Noodles & White-flour Pasta, Rice noodles. \\
\hline Breakfast cereals and related & All-Bran, Cornflakes, Oat bran, Muesli, Refined breakfast \\
\hline Products & Cereals, Wheat biscuits, Breakfast cereal bar. \\
\hline Bakery products & Cake, Muffin, Donut, Cupcake, Pancake, Croissant, Desserts. \\
\hline Dairy products and alternatives & $\begin{array}{l}\text { Cow and rice milk, Yoghurt, Ice cream, Soy-based product } \\
\text { alternatives. }\end{array}$ \\
\hline Fruit and fruit products & $\begin{array}{l}\text { Apricot, Peach, Banana, Orange, Mango, Pineapple, } \\
\text { Strawberry, Watermelon, Fig, Grape, Pear, Plum, Apple, } \\
\text { Mango, Fruit jams and preserves. }\end{array}$ \\
\hline Fruit juice & $\begin{array}{l}\text { 100\% fruit juices like Apple juice, Orange juice, Grapefruit } \\
\text { juice, Pineapple juice, Cranberry juice, Sugar sweetened } \\
\text { juices. }\end{array}$ \\
\hline Vegetables & Sweetcorn, Potato, Carrot, Pumpkin, Peas. \\
\hline $\begin{array}{l}\text { Snack products and } \\
\text { confectionery }\end{array}$ & $\begin{array}{l}\text { Milk chocolate, White chocolate, Chocolate hazelnut spread, } \\
\text { Candy bars, Fruits bar, Snack bar, Hard and gummy candies, } \\
\text { Brownies, Cookies, Popcorn, Potato crisps, Potato tortilla } \\
\text { chips, Corn chips. }\end{array}$ \\
\hline Sauce/Condiments & $\begin{array}{l}\text { Salad dressing, Dressing sauce for pasta other than tomato } \\
\text { sauce, Barbeque sauce, Ketchup, Mustard. }\end{array}$ \\
\hline Fructose, Glucose, Sucrose, Honey, Syrup. \\
\hline Combination foods & $\begin{array}{l}\text { Pizza, Focaccia, Panzerotto, White bread with } \\
\text { accompaniments, Quiche. }\end{array}$ \\
\hline Beverage & Sugar-sweetened beverages, sport drinks. \\
\hline
\end{tabular}


Supplemental Table 3. Baseline comparison of the two groups of the study population

\begin{tabular}{|c|c|c|c|}
\hline VARIABLES & $\begin{array}{c}\text { EXCELLENT } \\
(\mathrm{N}: 44)\end{array}$ & $\begin{array}{l}\text { POOR } \\
(\mathrm{N}: 63)\end{array}$ & $\mathbf{P}$ \\
\hline $\mathrm{M} / \mathrm{F}$ & $22 / 22$ & $29 / 34$ & NS \\
\hline AGE & 54 & 52 & NS \\
\hline $\mathrm{MS}+(\mathrm{T} 0)$ & $33 / 44(75 \%)$ & $42 / 63(67 \%)$ & NS \\
\hline $\mathrm{MS}+(\mathrm{T} 1)$ & $26 / 44(59 \%)$ & $36 / 63(57 \%)$ & NS \\
\hline CONTINUOS VARIABLES (BASELINE) & EXCELLENT & POOR & $\mathbf{P}$ \\
\hline $\mathrm{BW}, \mathrm{kg}$ & $94.5 \pm 2.8$ & $92.3 \pm 2.5$ & NS \\
\hline BMI, $\mathrm{kg} / \mathrm{m}^{2}$ & $34.0 \pm 0.9$ & $34.2 \pm 0.7$ & NS \\
\hline $\mathrm{WC}, \mathrm{cm}$ & $113.5 \pm 2.0$ & $112.6 \pm 1.8$ & NS \\
\hline $\mathrm{SBP}, \mathrm{mmHg}$ & $135.7 \pm 2.4$ & $133.0 \pm 1.7$ & NS \\
\hline $\mathrm{DBP}, \mathrm{mmHg}$ & $84.8 \pm 1.7$ & $83.6 \pm 1.2$ & NS \\
\hline TOT CHOL, mg/dl & $209.5 \pm 7.0$ & $197.7 \pm 4.7$ & NS \\
\hline HDL CHOL, mg/dl & $45.2 \pm 1.7$ & $46.9 \pm 1.3$ & NS \\
\hline LDL CHOL, mg/dl & $132.0 \pm 5.6$ & $120.5 \pm 4.2$ & NS \\
\hline $\mathrm{TG}, \mathrm{mg} / \mathrm{dl}$ & $171.6 \pm 13.7$ & $162.8 \pm 12.0$ & NS \\
\hline GLC, $\mathrm{mg} / \mathrm{dl}$ & $113.6 \pm 6.7$ & $112.6 \pm 5.0$ & NS \\
\hline HbA1c, \% & $6.3 \pm 0.3$ & $6.4 \pm 0.3$ & NS \\
\hline INSULIN, UI/ml & $24.1 \pm 5.2$ & $15.4 \pm 1.6$ & NS \\
\hline HOMA-IR & $7.8 \pm 2.2$ & $4.2 \pm 0.5$ & NS \\
\hline AST,U/L & $28.8 \pm 3.2$ & $25.7 \pm 2.5$ & NS \\
\hline ALT, U/L & $48.5 \pm 6.4$ & $46.4 \pm 4.3$ & NS \\
\hline CVR, \% & $7.4 \pm 1.4$ & $6.1 \pm 0.8$ & NS \\
\hline TREATMENTS & EXCELLENT & POOR & $\mathbf{P}$ \\
\hline Antidiabetics & $11(25 \%)$ & $18(29 \%)$ & NS \\
\hline Antihypertensive & $23(52 \%)$ & $35(56 \%)$ & NS \\
\hline Statins & $8(18 \%)$ & $15(24 \%)$ & NS \\
\hline Fibrates / PUFA $\Omega 3$ & $0(0 \%)$ & $5(8 \%)$ & 0.05 \\
\hline Physical exercise & $6(14 \%)$ & $12(19 \%)$ & NS \\
\hline
\end{tabular}

Data are reported as mean \pm standard error (SEM) or frequencies (percentages) for continuous and categorical variables, respectively. Comparisons of clinical and biochemical parameters among groups ("excellent" vs. "poor" adherence) are performed for baseline values with the Pearson $\mathrm{X}^{2}$ test and the Mann Whitney U test. Abbreviations: ALT, alanine transaminase; AST, aspartate transaminase; BMI, body mass index; BW, body weight; CVR, cardiovascular risk; DBP, diastolic blood pressure; GLC, fasting glucose; HbA1c, glycosylated hemoglobin; HDL CHOL, high-density lipoprotein cholesterol; HOMA-IR, homeostatic model assessment for insulin resistance; LDL CHOL, low-density lipoprotein cholesterol; PUFA, polyunsaturated fatty acids; SBP, systolic blood pressure; TG, triglyceride; TOT CHOL, total cholesterol; WC, waist circumference. 
Supplemental Table 4. Characteristics of the study population in the two groups before and after the MedLowC protocol in the "excellent" and "poor" adherence group.

\begin{tabular}{|l|c|c|c|c|c|c|}
\hline & \multicolumn{3}{|c|}{ Excellent adherence (N:44) } & \multicolumn{3}{c|}{ Poor adherence (N:63) } \\
\hline & Baseline & $\mathbf{6 ~ m o n t h s}$ & P & Baseline & 6 months & P \\
\hline BW, $\mathrm{kg}$ & $94.5 \pm 2.5$ & $85.5 \pm 2.5$ & $<0.001$ & $92.3 \pm 2.5$ & $90.7 \pm 2.5$ & 0.01 \\
\hline BMI, Kg/m ${ }^{2}$ & $34.0 \pm 0.9$ & $30.7 \pm 0.8$ & $<0.001$ & $34.2 \pm 0.7$ & $33.6 \pm 0.7$ & 0.01 \\
\hline WC, cm & $113.5 \pm 2$ & $105.7 \pm 1.8$ & $<0.001$ & $112.6 \pm 1.8$ & $111.0 \pm 1.8$ & NS $(0.07)$ \\
\hline SBP, mmHg & $135.7 \pm 2.4$ & $131.7 \pm 2.0$ & NS & $133.0 \pm 1.7$ & $130.5 \pm 1.7$ & NS \\
\hline DBP, mmHg & $84.8 \pm 1.7$ & $81.4 \pm 1.5$ & NS $(0.07)$ & $83.6 \pm 1.2$ & $82.6 \pm 1.3$ & NS \\
\hline TOT CHOL, mg/dl & $209.5 \pm 7.0$ & $179.8 \pm 4.4$ & $<0.001$ & $197.7 \pm 4.7$ & $197.7 \pm 4.8$ & NS \\
\hline HDL CHOL, mg/dl & $45.2 \pm 1.7$ & $47.7 \pm 1.6$ & NS $(0.06)$ & $46.9 \pm 1.3$ & $48.1 \pm 1.2$ & NS (0.08) \\
\hline LDL CHOL, mg/dl & $132.0 \pm 5.6$ & $111.8 \pm 4.6$ & $<0.001$ & $120.5 \pm 4.2$ & $122.2 \pm 4.0$ & NS \\
\hline TG, mg/dl & $171.6 \pm 13.7$ & $109.8 \pm 6.4$ & $<0.001$ & $162.8 \pm 12$ & $148.2 \pm 13.7$ & $\mathrm{NS}$ \\
\hline GLC, mg/dl & $113.6 \pm 6.7$ & $97.0 \pm 2.3$ & $<0.001$ & $112.6 \pm 5$ & $107.0 \pm 4.0$ & 0.02 \\
\hline HbA1c, \% & $6.3 \pm 0.3$ & $5.6 \pm 0.1$ & 0.003 & $6.4 \pm 0.3$ & $6.1 \pm 0.1$ & $\mathrm{NS}$ \\
\hline INSULIN, UI/ml & $24.1 \pm 5.2$ & $13.1 \pm 2.8$ & $<0.001$ & $15.4 \pm 1.6$ & $13.1 \pm 1.5$ & $\mathrm{NS}$ \\
\hline HOMA-IR & $7.8 \pm 2.2$ & $3.2 \pm 0.7$ & $<0.001$ & $4.2 \pm 0.5$ & $3.2 \pm 0.4$ & 0.04 \\
\hline AST, U/L & $28.8 \pm 3.2$ & $23.0 \pm 1.5$ & 0.04 & $25.7 \pm 2.5$ & $22.9 \pm 1.9$ & 0.01 \\
\hline ALT, U/L & $48.5 \pm 6.4$ & $30.6 \pm 3.0$ & 0.001 & $46.4 \pm 4.3$ & $36.6 \pm 4.0$ & 0.001 \\
\hline CVR, \% & $7.4 \pm 1.4$ & $6.3 \pm 1.1$ & 0.04 & $6.1 \pm 0.8$ & $5.4 \pm 0.7$ & NS (0.07) \\
\hline
\end{tabular}

Data are reported as mean \pm standard error (SEM) or frequencies (percentages) for continuous and categorical variables, respectively. Time dependent changes in continuous variables are assessed, separately in the two groups of adherence, with the non-parametric Wilcoxon Signed Rank Test. Abbreviations: ALT, alanine transaminase; AST, aspartate transaminase; BMI, body mass index; BW, body weight; CVR, cardiovascular risk; DBP, diastolic blood pressure; GLC, fasting glucose; HbA1c, glycosylated hemoglobin; HDL CHOL, high-density lipoprotein cholesterol; HOMA-IR, homeostatic model assessment for insulin resistance; LDL CHOL, low-density lipoprotein cholesterol; PUFA, polyunsaturated fatty acids; SBP, systolic blood pressure; TG, triglycerides; TOT CHOL, total cholesterol; WC, waist circumference. 
Supplemental Table 5. Multiple regression analysis of continuous variables with confounding factors.

\begin{tabular}{|c|c|c|c|c|}
\hline & \multicolumn{4}{|c|}{$\Delta \%$ POOR vs $\Delta \%$ EXCELLENT } \\
\hline & PHYSICAL & STATIN & ANTI-DIABETIC & FIBRATE/ PUFA $\Omega 3$ \\
\hline & ACTIVITY & THERAPY & THERAPY & THERAPY \\
\hline $\mathrm{BW}, \mathrm{kg}$ & NS (0.08) & NS & NS & NS \\
\hline BMI, $\mathrm{Kg} / \mathrm{m}^{2}$ & NS (0.08) & NS & NS & NS \\
\hline $\mathrm{WC}, \mathrm{cm}$ & NS & NS & $\mathrm{NS}$ & NS \\
\hline $\mathrm{SBP}, \mathrm{mmHg}$ & NS & NS & $\mathrm{NS}$ & NS \\
\hline $\mathrm{DBP}, \mathrm{mmHg}$ & NS & NS & NS & NS \\
\hline TOT CHOL, mg/dl & NS & 0.001 & $\mathrm{NS}$ & $\mathrm{NS}$ \\
\hline HDL CHOL, mg/dl & 0.05 & NS & $\mathrm{NS}$ & $\mathrm{NS}$ \\
\hline LDL CHOL, mg/dl & NS & NS (0.07) & $\mathrm{NS}$ & $\mathrm{NS}$ \\
\hline TG, mg/dl & NS & NS (0.09) & NS & NS \\
\hline GLC, mg/dl & NS & NS & NS (0.07) & NS \\
\hline $\mathrm{HbA1c}, \%$ & NS & NS (0.08) & 0.02 & NS \\
\hline INSULIN, UI/ml & NS & NS & NS & NS \\
\hline HOMA-IR & NS & NS & $\mathrm{NS}$ & NS \\
\hline AST, U/L & NS (0.09) & NS (0.08) & NS & NS \\
\hline ALT, U/L & NS & NS & $\mathrm{NS}$ & $\mathrm{NS}$ \\
\hline CVR, \% & NS & 0.05 & $\mathrm{NS}$ & $\mathrm{NS}$ \\
\hline
\end{tabular}

In order to compare the time dependent changes of each continuous variable between the two groups of adherence to the MedLowC protocol, we used a multiple regression analysis. This method provides a way of accounting for potentially confounding factors. Abbreviations: ALT, alanine transaminase; AST, aspartate transaminase; BMI, body mass index; BW, body weight; CVR, cardiovascular risk; DBP, diastolic blood pressure; GLC, fasting glucose; HbA1c, glycosylated hemoglobin; HDL CHOL, high-density lipoprotein cholesterol; HOMA-IR, homeostatic model assessment for insulin resistance; LDL CHOL, low-density lipoprotein cholesterol; SBP, systolic blood pressure; TG, triglyceride; TOT CHOL, total cholesterol; WC, waist circumference. 
Supplemental Table 6. Correlations between changes in $\mathrm{WC}(\Delta \%)$ and changes in patient biochemistry and clinical values $(\Delta \%)$.

\begin{tabular}{|l|c|c|}
\hline CONTINUOUS VARIABLE (overall) & R & P \\
\hline$\Delta \mathrm{BW}, \mathrm{kg}$ & 0.7 & $<0.001$ \\
\hline$\Delta \mathrm{BMI}, \mathrm{kg} / \mathrm{m}^{2}$ & 0.7 & $<0.001$ \\
\hline$\Delta \mathrm{HDL} \mathrm{CHOL}, \mathrm{mg} / \mathrm{dl}$ & -0.2 & $\mathrm{NS}(0.07)$ \\
\hline$\Delta \mathrm{GLC}, \mathrm{mg} / \mathrm{dl}$ & 0.2 & 0.03 \\
\hline$\Delta$ INSULIN, UI/ml & 0.4 & 0.01 \\
\hline$\Delta$ HOMA-IR & 0.4 & 0.005 \\
\hline$\Delta$ ALT, $\mathrm{U} / \mathrm{L}$ & 0.2 & $\mathrm{NS}(0.09)$ \\
\hline
\end{tabular}

The strength of the linear relationship between continuous variables was calculated with the Pearson's correlation coefficient. Abbreviations: ALT, alanine transaminase; BMI, body mass index; BW, body weight; GLC, fasting glucose; HDL CHOL, high-density lipoprotein cholesterol; HOMA-IR, homeostatic model assessment for insulin resistance.

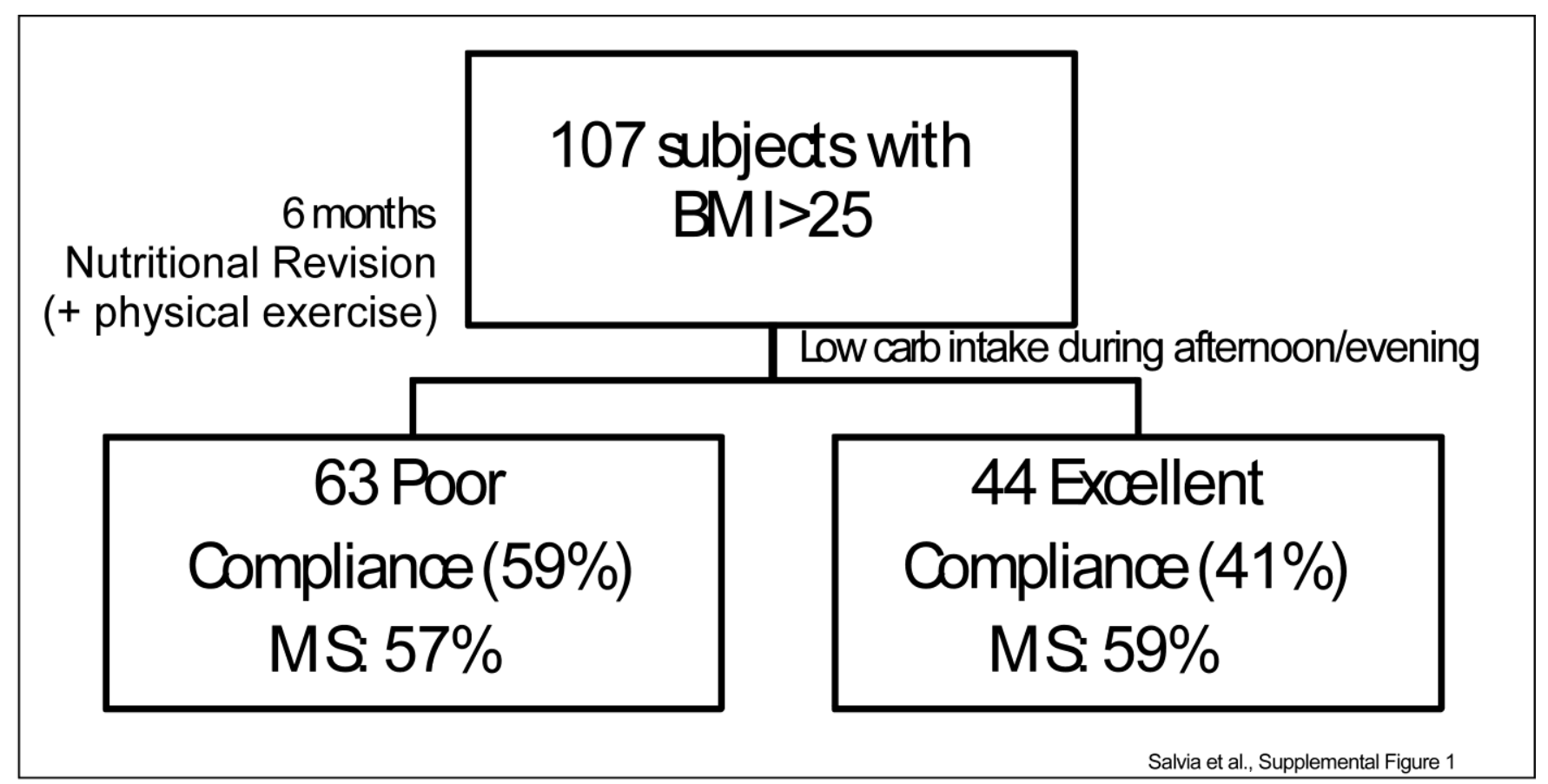

Supplemental Figure 1. Characterization of the study population. The flow chart shows the study population divided by the adherence to the unrestricted calorie Mediterranean diet with low carbohydrate intake in the evening. In the table, we characterized the same population according to their treatment for diabetes, hypercholesterolemia, hypertension and hypertriglyceridemia. 
Salvia et al., Online Supporting Materials

A

SBP
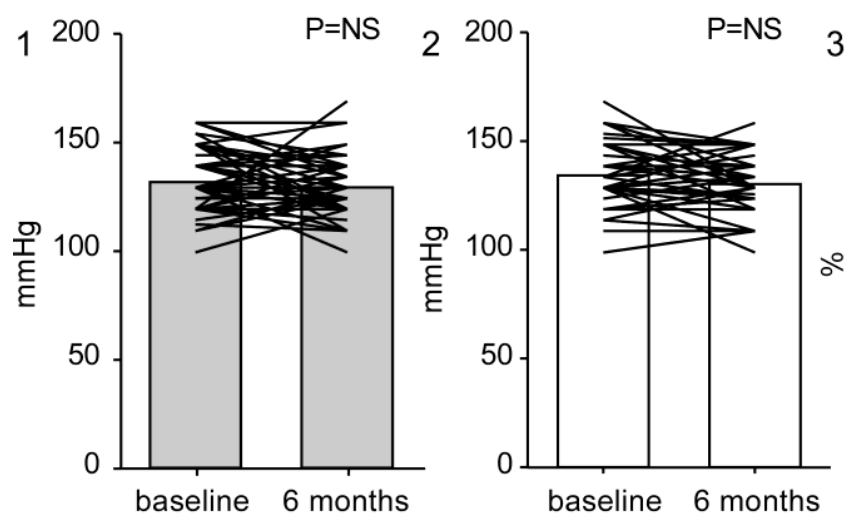

3

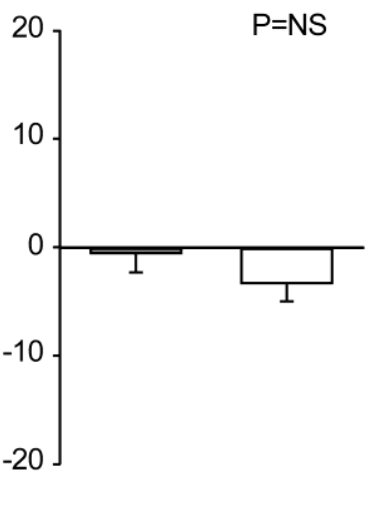

B

DBP
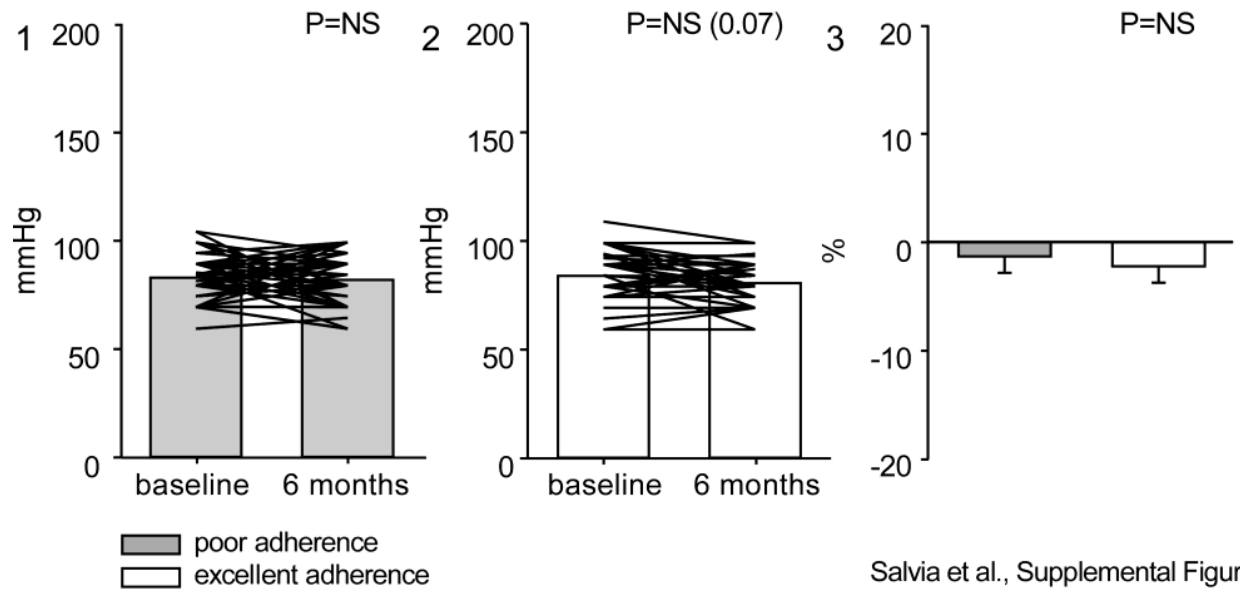

Salvia et al., Supplemental Figure 2

Supplemental Figure 2. Absence of changes in systolic (SBP, A) and diastolic blood pressure (DBP, B) following nutritional intervention. Diet had no impact on SBP and DBP compared to baseline values. Means are represented as bars: (grey) "poor" adherence group; (white) "excellent" adherence group. Lines represent patients (before/after). Error bars represent SEM. Time dependent changes in continuous variables were assessed with the nonparametric Wilcoxon Signed Rank Test ( $P$ value in section 1 and 2 of each variable plot). In order to compare the time dependent changes of each continuous variable between the two groups we used a multiple regression analysis (adjusted $\mathrm{P}$ value in section 3 of each variable plot; $\mathrm{P}$ value of the confounding variables investigated in Table S5).

\section{Supplemental Reference List}

\section{Reference List}

Panagiotakos DB, Pitsavos C, Stefanadis C.2006. Dietary patterns: a Mediterranean diet score and its relation to clinical and biological markers of cardiovascular disease risk. Nutr Metab Cardiovasc Dis 16:559-568. 